\title{
DECAY AT INFINITY OF CALORIC FUNCTIONS WITHIN CHARACTERISTIC HYPERPLANES
}

\author{
L. Escauriaza, C.E. Kenig, G. Ponce, and L. Vega
}

\begin{abstract}
It is shown that a function $u$ satisfying, $\left|\Delta u+\partial_{t} u\right| \leq M(|u|+|\nabla u|)$, $|u(x, t)| \leq M e^{M|x|^{2}}$ in $\mathbb{R}^{n} \times[0, T]$ and $|u(x, 0)| \leq C_{k} e^{-k|x|^{2}}$ in $\mathbb{R}^{n}$ for all $k \geq 1$, must vanish identically in $\mathbb{R}^{n} \times[0, T]$.
\end{abstract}

\section{Introduction}

E.M. Landis and O.A. Oleinik asked $[18, \S 4]$ for a proof of the following conjecture: If $u(x, t)$ is a bounded solution of a uniformly parabolic equation

$$
P u=\sum_{i, j=1}^{n} \partial_{i}\left(g^{i j}(x) \partial_{j} u\right)-\partial_{t} u+b(x) \cdot \nabla u+c(x) u=0,
$$

in the layer $\mathbb{R}^{n} \times[0, T]$ and the condition, $|u(x, T)| \leq N e^{-|x|^{2+\epsilon}}, x \in \mathbb{R}^{n}$, holds for some positive constants $N$ and $\epsilon$, then $u(x, t) \equiv 0$ in $\mathbb{R}^{n} \times[0, T]$.

As they wrote it, natural conditions should be placed on the behavior of the coefficients of $P$ at infinity for the conjecture to hold.

Here, we give an answer to this question when the leading parabolic operator is the backward heat operator and the lower order coefficients are bounded. In particular, we prove the following quantitative and qualitative results of unique continuation:

Theorem 1. Assume that a function u verifies the inequalities

$$
\left|\Delta u+\partial_{t} u\right| \leq M(|u|+|\nabla u|) \text { and }|u(x, t)| \leq M e^{M|x|^{2}} \text { in } \mathbb{R}^{n} \times[0, T] .
$$

Then, the following holds:

- If $\|u(\cdot, 0)\|_{L^{2}\left(B_{1}\right)}$ is positive, there is $N>0$ such that, when $|y| \geq N$

$$
\begin{aligned}
& \|u(\cdot, 0)\|_{L^{2}\left(B_{|y| / 2}(y)\right)} \geq e^{-N|y|^{2}} \text { and }\|u(\cdot, 0)\|_{L^{2}\left(B_{1}(y)\right)} \geq e^{-N|y|^{2} \log |y|} \text {. } \\
& \text { - } u \equiv 0 \text { in } \mathbb{R}^{n} \times[0, T] \text { if }|u(x, 0)| \leq C_{k} e^{-k|x|^{2}} \text { for all } x \in \mathbb{R}^{n} \text { and } k \geq 1 \text {. }
\end{aligned}
$$

Here, $B_{r}(y)=\left\{x \in \mathbb{R}^{n}:|x-y|<r\right\}$ and $B_{r}=B_{r}(0)$. We work with backward parabolic operators because it is more convenient in this context.

Received by the editors September 19, 2005.

The first and fourth authors are supported by MEC grant, MTM2004-03029 and by the European Commission via the network, Harmonic Analysis and Related Problems, project number RTN2-200100315. The second and third authors by NSF grants. 
When $n=1$ and provided that, $M^{-1} \leq \gamma(x) \leq M$ and $\left|\gamma^{\prime}(x)\right| \leq M$, the changes of variables

$$
y=\int_{0}^{x} \frac{d s}{\sqrt{\gamma(s)}}, v(x, t)=u(y, t)
$$

transform solutions $v$ of the inequalities

$$
\left|\partial_{x}\left(\gamma(x) \partial_{x} v\right)+\partial_{t} v\right| \leq M\left(|v|+\left|\partial_{x} v\right|\right),|v(x, t)| \leq M e^{M|x|^{2}} \text { in } \mathbb{R} \times[0, T],
$$

into solutions $u$ of backward parabolic inequalities, where the leading operator is the backward heat operator, as in (1.1). This and Theorem 1 prove the conjecture when $n=1$.

The first author, G. Seregin and V. Šverák proved in [9] the following qualitative property of unique continuation:

Let $\mathbb{R}_{+}^{n}=\left\{x=\left(x^{\prime}, x_{n}\right) \in \mathbb{R}^{n}: x_{n}>0\right\}$ and assume that $u$ satisfies

$$
\left|\Delta u+\partial_{t} u\right| \leq M(|u|+|\nabla u|), \quad|u(x, t)| \leq M e^{M|x|^{2}} \text { in } \mathbb{R}_{+}^{n} \times[0, T]
$$

and $u(x, 0)=0$ in $\mathbb{R}_{+}^{n}$. Then, $u \equiv 0$ in $\mathbb{R}_{+}^{n} \times[0, T]$.

This result is of interest in control theory; see [20], and as explained in [23] and [10], results of this type have shown to be helpful in the regularity theory for the Navier-Stokes equations. The arguments in the proof of Theorem 1 also imply the following improvement of the last result.

Theorem 2. Let $u$ verify (1.2) and set $e_{n}=(0, \ldots, 0,1)$. Then, the following holds:

- If $\|u(\cdot, 0)\|_{L^{2}\left(B_{1}\left(4 e_{n}\right)\right)}$ is positive, there is $N>0$ such that, when $y \geq N$

$$
\begin{gathered}
\|u(\cdot, 0)\|_{L^{2}\left(B_{y / 2}\left(y e_{n}\right)\right)} \geq e^{-N y^{2}} \text { and }\|u(\cdot, 0)\|_{L^{2}\left(B_{1}\left(y e_{n}\right)\right)} \geq e^{-N y^{2} \log y} . \\
\text { - } u \equiv 0 \text { in } \mathbb{R}_{+}^{n} \times[0, T] \text { if }|u(x, 0)| \leq C_{k} e^{-k|x|^{2}} \text { for all } x \in \mathbb{R}_{+}^{n} \text { and } k \geq 1 .
\end{gathered}
$$

We present in sections 2 and 3 two different proofs of Theorems 1 . The first one is based on Carleman inequality methods while the second on frequency function methods. The main tools in both proofs are a rescaling argument and a quantification of the size of the constants involved in the two sphere and one cylinder inequalities satisfied by solutions of certain parabolic equations, in terms of the $L^{\infty}$-norm of the lower order coefficients and of the time of existence of solutions. See [2, Lemma 3.10], where similar ideas appeared but dealing with three sphere inequalities and elliptic equations. In section 4, we outline the proof of Theorem 2 .

With the purpose of simplifying the arguments below, we only prove Theorems 1 and 2, when the growth condition in (1.1) or (1.2), $|u(x, t)| \leq M e^{M|x|^{2}}$, is replaced by $u$ is bounded. The interested reader can easily verify that the arguments below can be adapted to the more general case.

\section{First Proof of Theorem 1}

The next five Lemmas are used in the first proof of Theorem 1. The first one, Lemma 1 , is in a certain sense a localized version of the standard energy inequality satisfied by solutions of parabolic inequalities (See [7, Lemmas 1 and 5] for other versions of this Lemma). The Lemmas 2 and 3 appeared in [7, Lemmas 2, 3]. 
Lemma 1. Assume that $u$ satisfies, $\left|\Delta u+\partial_{t} u\right| \leq R^{2}|u|+R|\nabla u|,\|u\|_{\infty} \leq 1,\|\nabla u\|_{\infty} \leq$ $R$ in $B_{4} \times\left[0, \frac{1}{R^{2}}\right]$ and $\|u(\cdot, 0)\|_{L^{2}\left(B_{\rho}\right)} \geq \theta R^{-n / 2}$ for some $\theta, \rho$ in $(0,1]$ and $R>0$. Then, there is $N=N(n, \theta)$ such that the inequality, $\sqrt{N}\|u(\cdot, t)\|_{L^{2}\left(B_{2 \rho}\right)} \geq R^{-n / 2}$ holds, when $0<t \leq 1 / R^{2}$ and $R>N / \rho$.

Proof. Assume first that $\rho=1$ and set $f=u \varphi$, where $\varphi \in C_{0}^{\infty}\left(B_{2}\right), 0 \leq \varphi \leq 1$ and $\varphi=1$ in $B_{3 / 2}$. Then,

$$
\left|\Delta f+\partial_{t} f\right| \leq R^{2}|f|+R|\nabla f|+N R \chi_{B_{2} \backslash B_{3 / 2}} .
$$

Setting $H(t)=\int f^{2}(x, t) G(x-y, t) d x$, where $G(x, t)=t^{-n / 2} e^{-|x|^{2} / 4 t}$ and $y \in B_{1}$, we have

$$
\dot{H}(t)=2 \int f\left(\Delta f+\partial_{t} f\right) G(x-y, t) d x+2 \int|\nabla f|^{2} G(x-y, t) d x,
$$

and from (2.1), (2.2) and the Cauchy-Schwarz's inequality

$$
\dot{H}(t) \geq-8 R^{2} H(t)-N e^{-1 / N t} .
$$

Integration of this inequality in $(0, t), 0<t \leq \frac{1}{R^{2}}$, gives

$$
N \int f^{2}(x, t) G(x-y, t) d x \geq u^{2}(y, 0)-N e^{-1 / N t} .
$$

Integrating the last inequality over $B_{1}$ and recalling that $\int G(x-y, t) d y=1$, we get

$$
N \int_{B_{2}} u^{2}(x, t) d x \geq \int_{B_{1}} u^{2}(x, 0) d x-N e^{-1 / N t} \geq R^{-n}\left(\theta^{2}-N e^{-R^{2} / 2 N}\right),
$$

when $0<t \leq 1 / R^{2}$, which implies Lemma 1 when $\rho=1$.

When $\rho$ is in $(0,1)$, the function $u_{\rho}(x, t)=u\left(\rho x, \rho^{2} t\right)$, satisfies the conditions in Lemma 1 with $\rho=1$ and $R$ replaced by $\rho R$. The Lemma then, follows after rescaling to the case $\rho=1$.

Lemma 2. The inequality

$$
\int \frac{|x|^{2}}{8 a} h^{2} e^{-|x|^{2} / 4 a} d x \leq 2 a \int|\nabla h|^{2} e^{-|x|^{2} / 4 a} d x+\frac{n}{2} \int h^{2} e^{-|x|^{2} / 4 a} d x
$$

holds for all $h \in C_{0}^{\infty}\left(\mathbb{R}^{n}\right)$ and $a>0$.

Proof. The inequality follows setting $v=h e^{-|x|^{2} / 8 a}$ and from the identity

$$
\begin{gathered}
2 a \int|\nabla h|^{2} e^{-|x|^{2} / 4 a} d x+\frac{n}{2} \int h^{2} e^{-|x|^{2} / 4 a} d x-\int \frac{|x|^{2}}{8 a} h^{2} e^{-|x|^{2} / 4 a} d x \\
=2 a \int|\nabla v|^{2} d x .
\end{gathered}
$$

Lemma 3. Assume that $N$ and $\Theta$ verify $N \log (N \Theta) \geq 1, h \in C_{0}^{\infty}\left(\mathbb{R}^{n}\right)$ and that the inequality

$$
2 a \int|\nabla h|^{2} e^{-|x|^{2} / 4 a} d x+\frac{n}{2} \int h^{2} e^{-|x|^{2} / 4 a} d x \leq N \log (N \Theta) \int h^{2} e^{-|x|^{2} / 4 a} d x
$$


holds, when $0<a \leq \frac{1}{12 N \log (N \Theta)}$. Then,

$$
\int_{B_{2 r}} h^{2} d x \leq(N \Theta)^{N} \int_{B_{r}} h^{2} d x \text {, when } 0<r \leq 1 / 2 .
$$

Proof. The inequality satisfied by $h$ and Lemma 2 show that

$$
\int \frac{|x|^{2}}{8 a} h^{2} e^{-|x|^{2} / 4 a} d x \leq N \log (N \Theta) \int h^{2} e^{-|x|^{2} / 4 a} d x,
$$

when $a \leq 1 /(12 N \log (N \Theta))$. For given $0<r \leq 1 / 2$ and $0<a \leq \frac{r^{2}}{16 N \log (N \Theta)}$, the last inequality implies

$$
\begin{gathered}
\int \frac{|x|^{2}}{8 a} h^{2} e^{-|x|^{2} / 4 a} d x \leq N \log (N \Theta)\left[\int_{B_{r}} h^{2} d x+\frac{8 a}{r^{2}} \int_{\mathbb{R}^{n} \backslash B_{r}} \frac{|x|^{2}}{8 a} h^{2} e^{-|x|^{2} / 4 a} d x\right] \\
\leq N \log (N \Theta) \int_{B_{r}} h^{2} d x+\frac{1}{2} \int \frac{|x|^{2}}{8 a} h^{2} e^{-|x|^{2} / 4 a} d x .
\end{gathered}
$$

Thus,

$$
\int \frac{|x|^{2}}{16 a} h^{2} e^{-|x|^{2} / 4 a} d x \leq N \log (N \Theta) \int_{B_{r}} h^{2} d x
$$

when $0<a \leq r^{2} / 16 N \log (N \Theta)$. Now,

$$
e^{-|x|^{2} / 4 a}|x|^{2} /(16 a) \geq(N \Theta)^{-N} N \log (N \Theta),
$$

when $r \leq|x| \leq 2 r$ and $a=r^{2} / 16 N \log (N \Theta)$. This and (2.3) imply

$$
\int_{B_{2 r}} h^{2} d x \leq(N \Theta)^{N} \int_{B_{r}} h^{2} d x, \text { when } 0<r \leq 1 / 2 .
$$

The Lemma 4 contains the Carleman inequality we need. Here, $d X=d x d t$ is the Lebesgue measure in $\mathbb{R}_{+}^{n+1}$ and $\sigma_{a}(t)=\sigma(t+a)$, denotes the translation by $a>0$ of a function $\sigma$ of the time-variable.

Lemma 4. Given $\alpha \geq 2+n / 2$, there are $N=N(n)$ and an increasing function, $\sigma:[0,+\infty) \longrightarrow[0,+\infty)$ verifying, $t / N \leq \sigma(t) \leq t$ in $[0,4 / \alpha]$ and such that the inequality

$$
\begin{gathered}
\alpha^{2} \int \sigma_{a}^{-\alpha} f^{2} e^{-|x|^{2} / 4(t+a)} d X+\alpha \int \sigma_{a}^{1-\alpha}|\nabla f|^{2} e^{-|x|^{2} / 4(t+a)} d X \\
\leq N \int \sigma_{a}^{1-\alpha}\left(\Delta f+\partial_{t} f\right)^{2} e^{-|x|^{2} / 4(t+a)} d X \\
+\sigma(a)^{-\alpha}\left[-(a / N) \int|\nabla f(x, 0)|^{2} e^{-|x|^{2} / 4 a} d x+N \alpha \int f^{2}(x, 0) e^{-|x|^{2} / 4 a} d x\right]
\end{gathered}
$$

holds, when $0<a \leq \frac{1}{\alpha}$ and $f \in C_{0}^{\infty}\left(\mathbb{R}^{n} \times\left[0, \frac{4}{\alpha}\right)\right)$.

This inequality appeared first in $[12, \S 3]$ in the context of variable coefficients parabolic operators. The inequality is not stated there as it is shown above, some additional terms appear or are missing on the right hand side of the corresponding inequality in $[12, \S 3]$. These additional terms arise from the purpose of controlling 
certain error terms generated by the variable coefficients of the parabolic operator, and they can be dropped when the operator is the backward heat operator. Other versions of this inequality appeared in $[8,(1.4)]$, [10, Proposition 6.1$]$ and $[9, \S 3]$, but none of them is stated or proved as we need need it here.

As it is usual in the context of $L^{2}$-Carleman estimates, we use suitable integration by parts to prove Lemma 4 . The calculations can be organized either by using identities developed in [6, Lemma 1] and [8, Lemma 3], or by following more or less standard calculations with new dependent variables and commutators in the spirit of [15], [16] or [25]. In this paper we will use the former method.

Proof. Assume first that the following claim holds:

There are $N=N(n)$ and an increasing function, $h:[0,+\infty) \longrightarrow[0,+\infty)$, verifying, $t / N \leq h(t) \leq t$ in $[0,6]$ and such that the inequality

$$
\begin{gathered}
\alpha \int h_{a}^{-\alpha} u^{2} e^{-|x|^{2} / 4(t+a)} d X+\int h_{a}^{1-\alpha}|\nabla u|^{2} e^{-|x|^{2} / 4(t+a)} d X \\
\leq N \int h_{a}^{1-\alpha}\left(\Delta u+\partial_{t} u\right)^{2} e^{-|x|^{2} / 4(t+a)} d X \\
+h(a)^{-\alpha}\left[-(a / N) \int|\nabla u(x, 0)|^{2} e^{-|x|^{2} / 4 a} d x+N \alpha \int u^{2}(x, 0) e^{-|x|^{2} / 4 a} d x\right]
\end{gathered}
$$

holds, when $\alpha \geq 2+n / 2,0<a \leq 1$ and $u \in C_{0}^{\infty}\left(\mathbb{R}^{n} \times[0,4)\right)$.

Take as $u$ in (2.4) the function, $u(x, t)=f(x / \sqrt{\alpha}, t / \alpha)$, when $f \in C_{0}^{\infty}\left(\mathbb{R}^{n} \times\left[0, \frac{4}{\alpha}\right)\right)$ and define $\sigma(t)=h(\alpha t) / \alpha$. Then, it is simple to verify that Lemma 4 holds after undoing the change of variables and counting of the number of $\alpha$ 's at each side of the inequality.

In order to prove the claim we recall the following identity $[8,(2.4)]$, which holds when $\alpha \in \mathbb{R}, u \in C_{0}^{\infty}\left(\mathbb{R}^{n} \times[0,4)\right), G$ is a positive caloric function in $\mathbb{R}_{+}^{n+1}$ and $\gamma:[0,+\infty) \longrightarrow(0,+\infty)$ is an increasing smooth function:

$$
\begin{gathered}
\frac{2 \gamma^{1-\alpha}}{\dot{\gamma}}\left(\partial_{t} u-\nabla \log G \cdot \nabla u-\frac{\alpha \dot{\gamma}}{2 \gamma} u\right)^{2} G+\frac{\gamma^{1-\alpha}}{\dot{\gamma}} \mathcal{D}_{G} \nabla u \cdot \nabla u G \\
=\frac{2 \gamma^{1-\alpha}}{\dot{\gamma}}\left(\partial_{t} u-\nabla \log G \cdot \nabla u-\frac{\alpha \dot{\gamma}}{2 \gamma} u\right)\left(\Delta u+\partial_{t} u\right) G \\
\quad+\partial_{t}\left[\frac{\gamma^{1-\alpha}}{\dot{\gamma}}|\nabla u|^{2} G-\frac{\alpha \gamma^{-\alpha}}{2} u^{2} G\right] \\
+\frac{\gamma^{1-\alpha}}{\dot{\gamma}} \nabla \cdot\left[2 \partial_{t} u G \nabla u+|\nabla u|^{2} \nabla G-2(\nabla G \cdot \nabla u) \nabla u-\frac{\alpha \dot{\gamma}}{\gamma} u G \nabla u-\frac{\alpha \dot{\gamma}}{2 \gamma} u^{2} \nabla G\right] .
\end{gathered}
$$

Here, $\mathcal{D}_{G}$ denotes the $n \times n$ matrix

$$
\mathcal{D}_{G}=\overbrace{\log \left(\frac{\gamma}{\dot{\gamma}}\right)} \mathcal{I}+2 D^{2}(\log G) \text {. }
$$

If in (2.5) we set $\gamma(t)=h_{a}(t)$, where $h(t)=t e^{-t / 6}, a \in(0,1]$ and let $G$ be the translated Gauss Kernel, $G_{a}(x, t)=(t+a)^{-n / 2} e^{-|x|^{2} / 4(t+a)}$, we have

$$
\frac{1}{e}(t+a) \leq h_{a}(t) \leq t+a, \frac{1}{6 e} \leq \dot{h}_{a}(t) \leq 1 \text { and } \mathcal{D}_{G_{a}} \geq \frac{1}{6} \mathcal{I}, \text { when } t \in(0,4] .
$$

Integrating the identity (2.5) over $\mathbb{R}_{+}^{n+1}$, one gets from (2.6) and the Cauchy-Schwarz inequality (which is used to handle the first integral on the right hand side of the 
formula (2.5)), the bound

$$
\begin{gathered}
\int h_{a}^{1-\alpha}|\nabla u|^{2} G_{a} d X \leq N \int h_{a}^{1-\alpha}\left(\Delta u+\partial_{t} u\right)^{2} G_{a} d X+h(a)^{-\alpha-n / 2} \times \\
{\left[-(a / N) \int|\nabla u(x, 0)|^{2} e^{-|x|^{2} / 4 a} d x+N \alpha \int u^{2}(x, 0) e^{-|x|^{2} / 4 a} d x\right] .}
\end{gathered}
$$

Finally, the claim follows after multiplication of the identity

$$
\left(\Delta+\partial_{t}\right)\left(u^{2}\right)=2 u\left(\Delta u+\partial_{t} u\right)+2|\nabla u|^{2}
$$

by $h_{a}^{1-\alpha} G_{a}$, the integration by parts of the operator $\Delta+\partial_{t}$, which is acting on $u^{2}$ over the other terms in the corresponding integral over $\mathbb{R}^{n} \times[0,4)$ and using the Cauchy-Schwarz inequality to handle the cross term, (2.6) and (2.7).

Lemma 5. Given $\theta \in(0,1]$, there are $N=N(n, \theta) \geq 1$ and $\rho=\rho(n, \theta)$ in $(0,1]$ such that the following holds:

If $u$ satisfies $\left|\Delta u+\partial_{t} u\right| \leq R^{2}|u|+R|\nabla u|,\|u\|_{\infty} \leq 1,\|\nabla u\|_{\infty} \leq R$ in $B_{4} \times\left[0, \frac{1}{R^{2}}\right]$ and $\|u(\cdot, 0)\|_{L^{2}\left(B_{\rho}\right)} \geq \theta R^{-n / 2}$. Then,

- $\left\|u(\cdot, 0) e^{-R^{2}|x|^{2} / \epsilon}\right\|_{L^{2}\left(B_{4}\right)} \geq e^{-N R^{2} \log \left(\frac{1}{\epsilon}\right)}$, when $0<\epsilon \leq \frac{1}{3 N}, R \geq N$.

- $\|u(\cdot, 0)\|_{L^{2}\left(B_{r}\right)} \geq e^{-N R^{2} \log \left(\frac{N}{r}\right)}$, when $0<r \leq \frac{1}{2}, R \geq N$.

Proof. In Lemma 4, take as $f$, the function, $f=u \varphi(x) \psi(t)$, where $\varphi \in C_{0}^{\infty}\left(B_{4}\right)$, $0 \leq \varphi \leq 1, \varphi=1$ in $B_{3}$ and $\varphi=0$ outside $B_{\frac{7}{2}}, \psi=1$ when $0<t \leq \frac{1}{\alpha}$ and $\psi=0$ when $t \geq \frac{2}{\alpha}$. Then,

$$
\left|\Delta f+\partial_{t} f\right| \leq R^{2}|f|+R|\nabla f|+N(\alpha+R) \chi_{B_{4} \times\left[0, \frac{2}{\alpha}\right] \backslash B_{3} \times\left[0, \frac{1}{\alpha}\right]} .
$$

The facts that, $t / N \leq \sigma(t) \leq t$ on $\left[0, \frac{6}{\alpha}\right]$, that $\sigma_{a}^{1-\alpha} G_{a} \leq N^{\alpha+\frac{n}{2}} \alpha^{\alpha+\frac{n}{2}-1}$ in the region $B_{4} \times\left[0, \frac{2}{\alpha}\right] \backslash B_{3} \times\left[0, \frac{1}{\alpha}\right],(2.8)$ and standard arguments with Carleman inequalities, imply the estimate

$$
\begin{aligned}
& \alpha^{2} \int_{0}^{\frac{1}{\alpha}} \int_{B_{2}}(t+a)^{-\alpha} u^{2} e^{-|x|^{2} / 4(t+a)} d X \leq N^{\alpha} \alpha^{\alpha+1}+N^{\alpha} \sigma(a)^{-\alpha} \times \\
& {\left[-(a / N) \int|\nabla f(x, 0)|^{2} e^{-|x|^{2} / 4 a} d x+N \alpha \int f^{2}(x, 0) e^{-|x|^{2} / 4 a} d x\right], }
\end{aligned}
$$

when $\alpha \geq N R^{2}$ and $0<a \leq \frac{1}{\alpha}$.

For $\rho$ in $(0,1]$, which will be chosen later and Lemma 1, we know that

$$
\sqrt{N}\|u(\cdot, t)\|_{L^{2}\left(B_{2 \rho}\right)} \geq R^{-n / 2} \text {, when } 0<t \leq 1 / R^{2} \text { and } R>N / \rho .
$$

This and the conditions, $0<a \leq \frac{\rho^{2}}{2 \alpha}$ and $\alpha \geq N R^{2}$, imply that the left hand side of (2.9) is bounded from below by

$$
\alpha^{2} \int_{\frac{\rho^{2}}{2 \alpha}-a}^{\frac{\rho^{2}}{\alpha}-a} \int_{B_{2 \rho}}(t+a)^{-\alpha} e^{-\frac{\rho^{2}}{(t+a)}} u^{2} d X \geq \frac{\alpha^{\alpha+1} \rho^{2}}{2 N R^{n}}\left(\frac{1}{\rho e}\right)^{2 \alpha} .
$$

Inequalities (2.9) and (2.10) show, that to make sure that the left hand side of (2.9) is larger than four times the first term on right hand side of (2.9), when $\alpha \geq N R^{2}$ 
and $0<a \leq \frac{\rho^{2}}{2 \alpha}$, it suffices to know that

$$
\left(\frac{1}{\rho e}\right)^{2 \alpha} \geq 8 N^{\alpha+1} R^{n} / \rho^{2}
$$

Choose then $\rho$ as the solution of the equation $\frac{1}{\rho e}=\sqrt{8 N}$. Then, (2.11) holds when $8^{\alpha-1} \geq N R^{n} / \rho^{2}$. Thus, there are fixed constants, $\rho=\rho(n, \theta)$ in $(0,1]$ and $N=$ $N(n, \theta) \geq 1$ such that, under the conditions in Lemma 5 , we have

$$
\begin{aligned}
& \frac{1}{2} \int_{0}^{\frac{1}{\alpha}} \int_{B_{2}}(t+a)^{-\alpha} u^{2} e^{-|x|^{2} / 4(t+a)} d X+N^{\alpha} \alpha^{\alpha+1} \leq N^{\alpha} \sigma(a)^{-\alpha} \times \\
& {\left[-(a / N) \int|\nabla f(x, 0)|^{2} e^{-|x|^{2} / 4 a} d x+N \alpha \int f^{2}(x, 0) e^{-|x|^{2} / 4 a} d x\right], }
\end{aligned}
$$

when $R \geq N, \alpha \geq N R^{2}$ and $0<a \leq \frac{\rho^{2}}{12 \alpha}$. In particular, there is $N=N(n, \theta)$ such that

$$
\begin{array}{rl}
N^{-\alpha} \alpha^{\alpha+1} a^{\alpha} \leq-(a / N) \int|\nabla f(x, 0)|^{2} e^{-|x|^{2} / 4 a} & d x \\
& +N \alpha \int f^{2}(x, 0) e^{-|x|^{2} / 4 a} d x,
\end{array}
$$

when $R \geq N, \alpha=N R^{2}$ and $0<a \leq 1 / 12 N R^{2}$.

Recalling the definition of $f$, choose $a=\frac{\epsilon}{8 R^{2}}$ in (2.12). It implies the inequality

$$
e^{-2 N R^{2} \log \left(\frac{1}{\epsilon}\right)} \leq \int_{B_{4}} u^{2}(x, 0) e^{-2 R^{2}|x|^{2} / \epsilon} d x, \text { when } 0<\epsilon \leq \frac{2}{3 N}, R \geq N
$$

and proves the first claim in Lemma 5. The inequality (2.12) also implies the bound

$$
\begin{aligned}
& 2 a \int|\nabla f(x, 0)|^{2} e^{-|x|^{2} / 4 a} d x+\frac{n}{2} \int f^{2}(x, 0) e^{-|x|^{2} / 4 a} d x \\
& \leq N R^{2} \int f^{2}(x, 0) e^{-|x|^{2} / 4 a} d x \quad, \quad \text { when } 0<a \leq \frac{1}{12 N R^{2}}, R \geq N .
\end{aligned}
$$

From Lemma 3 with $h=f(\cdot, 0)$ and the above estimate, we obtain

$$
\int_{B_{2 r}} u^{2}(x, 0) d x \leq e^{N R^{2}} \int_{B_{r}} u^{2}(x, 0) d x, \text { when } 0<r \leq 1 / 2, R \geq N .
$$

For these values of $r$, choose $k \geq 2$ such that, $2^{-k}<r \leq 2^{-k+1}$ and iterate (2.13), when $r=2^{-j}, j=0, \ldots, k-1$. It gives

$$
\int_{B_{1}} u^{2}(x, 0) d x \leq e^{2 N R^{2} \log (1 / r)} \int_{B_{r}} u^{2}(x, 0) d x, \text { when } 0<r \leq 1 / 2, R \geq N,
$$

which proves the second claim.

Proof of Theorem 1. Without loss of generality we may assume that $u$ satisfies

$$
\left|\Delta u+\partial_{t} u\right| \leq|u|+|\nabla u| \text { and }|u| \leq 1 \text { in } \mathbb{R}^{n} \times[0,4] .
$$


Choose $\theta$ in $(0,1]$ such that, $\theta \leq\|u(\cdot, 0)\|_{L^{2}\left(B_{1}\right)}$. If $\rho$ is the constant associated to $\theta$ in Lemma 5 , define $u_{R}(x, t)=u\left(R x+y, R^{2} t\right)$, when $R \rho=2|y|$ is large, $y \in \mathbb{R}^{n}$. Then,

$$
R^{n / 2}\left\|u_{R}(\cdot, 0)\right\|_{L^{2}\left(B_{\rho}\right)}=\|u(\cdot, 0)\|_{L^{2}\left(B_{2|y|}(y)\right)} \geq\|u(\cdot, 0)\|_{L^{2}\left(B_{1}\right)} \geq \theta
$$

and the standard interior estimates for solutions to parabolic equations [17] show that $u_{R}$ satisfies the conditions in Lemma 5. The first claim in Lemma 5 applied to $u_{R}$ and the change of variables, $R x+y=z$, give that for $\epsilon$ sufficiently small

$$
\begin{aligned}
& R^{n / 2} e^{-N R^{2} \log \left(\frac{1}{\epsilon}\right)} \leq\left\|u(\cdot, 0) e^{-|x-y|^{2} / \epsilon}\right\|_{L^{2}\left(B_{4|y| / \rho}(y)\right)} \\
& \quad \leq\|u(\cdot, 0)\|_{L^{2}\left(B_{|y| / 2}(y)\right)}+R^{n / 2} e^{-R^{2} / 8 \epsilon}, \text { when } 0<\epsilon \leq \frac{1}{N}, R \geq N,
\end{aligned}
$$

and choosing $\epsilon$ small in (2.14), implies the first inequality in Theorem 1.

The second claim in Lemma 5 applied to $u_{R}$, with $r=1 / R$ and the same change of variables, give

$$
R^{-n / 2}\|u(\cdot, 0)\|_{L^{2}\left(B_{1}(y)\right)}=\left\|u_{R}(\cdot, 0)\right\|_{L^{2}\left(B_{1 / R}\right)} \geq e^{-N R^{2} \log (N R)},
$$

which proves the second inequality in Theorem 1 .

What has been proved so far, shows that the condition

$$
|u(x, 0)| \leq C_{k} e^{-k|x|^{2}} \text { for all } x \in \mathbb{R}^{n} \text { and } k \geq 1,
$$

can only hold when $\|u(\cdot, 0)\|_{L^{2}\left(B_{1}\right)}$ vanishes. The results in [1] or [12, Theorem 3] prove that the latter is only possible, when $u(\cdot, 0) \equiv 0$. Then, standard backward uniqueness arguments for parabolic equations imply, $u \equiv 0$ in $\mathbb{R}^{n} \times[0,4]$, when $u \in$ $L^{\infty}\left(0,4 ; L^{2}\left(\mathbb{R}^{n}\right)\right)$ [11]. If one wants to relax the latter condition and to allow $u$ to grow as a quadratic exponential at infinity in the layer $\mathbb{R}^{n} \times[0,4]$, the fact that $u \equiv 0$ in $\mathbb{R}^{n} \times[0,4]$ follows from the arguments in $[12$, Theorem 3$]$ or the Carleman inequality $(2.4)$.

\section{Second Proof of Theorem 1}

The second proof of Theorem 1 is based in Lemmas 6 and 2 .

Lemma 6. Given $a>0$ and $f \in W^{2, \infty}\left(\mathbb{R}_{+}^{n+1}\right)$, set

$$
\begin{gathered}
H_{a}(t)=\int_{\mathbb{R}^{n}} f^{2} G_{a} d x, D_{a}(t)=\int_{\mathbb{R}^{n}}|\nabla f|^{2} G_{a} d x \text { and } N_{a}(t)=\frac{2(t+a) D_{a}(t)}{H_{a}(t)}, \\
\text { where } G_{a}(x, t)=(t+a)^{-n / 2} e^{-|x|^{2} / 4(t+a)} \text {. Then, } \\
\dot{N}_{a}(t) \geq-\frac{(t+a)}{H_{a}(t)} \int\left(\Delta f+\partial_{t} f\right)^{2} G_{a} d x
\end{gathered}
$$

The monotonicity results implied by this Lemma (e.g. $N_{a}(t)$ is nondecreasing when $f$ is a backward caloric in $R_{+}^{n+1}$ ) are within the category of what in the literature have been called frequency function arguments. The frequency function here is $N_{a}(t)$. This frequency function seems to have first appeared or been used in the context of unique continuation for parabolic equations in [21], when $a=0$ and in [12], when 
$a>0$. Related results, though with perhaps different purposes, appeared in [13] and $[14]$.

The next proof of Lemma 6 comes from [7, Lemma 2].

Proof. The identities $\partial_{t} G_{a}-\Delta G_{a}=0, \nabla G_{a}=-\frac{x}{2(t+a)} G_{a}, \Delta=\operatorname{div}(\nabla)$ and integration by parts imply the following identities

$$
\begin{aligned}
& \dot{H}_{a}(t)=2 \int f\left(\Delta f+\partial_{t} f\right) G_{a} d x+2 D_{a}(t), \\
\dot{H}_{a}(t) & =2 \int f\left(\partial_{t} f+\frac{x}{2(t+a)} \cdot \nabla f-\frac{1}{2}\left(\Delta f+\partial_{t} f\right)\right) G_{a} d x \\
& +\int f\left(\Delta f+\partial_{t} f\right) G_{a} d x \\
D_{a}(t) & =\int f\left(\partial_{t} f+\frac{x}{2(t+a)} \cdot \nabla f-\frac{1}{2}\left(\Delta f+\partial_{t} f\right)\right) G_{a} d x \\
& -\frac{1}{2} \int f\left(\Delta f+\partial_{t} f\right) G_{a} d x
\end{aligned}
$$

and

$$
\begin{aligned}
\dot{H}_{a}(t) D_{a}(t) & =2\left(\int f\left(\partial_{t} f+\frac{x}{2(t+a)} \cdot \nabla f-\frac{1}{2}\left(\Delta f+\partial_{t} f\right)\right) G_{a} d x\right)^{2} \\
& -\frac{1}{2}\left(\int f\left(\Delta f+\partial_{t} f\right) G_{a} d x\right)^{2} .
\end{aligned}
$$

The Rellich-Něcas identity with vector field $\nabla G_{a}$

$$
\begin{aligned}
\operatorname{div}\left(\nabla G_{a}|\nabla f|^{2}\right) & -2 \operatorname{div}\left(\left(\nabla f \cdot \nabla G_{a}\right) \nabla f\right) \\
& =\Delta G_{a}|\nabla f|^{2}-2 D^{2} G_{a} \nabla f \cdot \nabla f-2 \nabla f \cdot \nabla G_{a} \Delta f
\end{aligned}
$$

and integration by parts give

$$
\begin{aligned}
& \int \Delta G_{a}|\nabla f|^{2} d x=2 \int D^{2} G_{a} \nabla f \cdot \nabla f d x+2 \int \nabla f \cdot \nabla G_{a} \Delta f d x \\
= & 2 \int\left(\frac{x}{2(t+a)} \cdot \nabla f\right)^{2} G_{a} d x-2 \int \frac{x}{2(t+a)} \cdot \nabla f \Delta f G_{a} d x-D_{a}(t) /(t+a) .
\end{aligned}
$$

Again, the fact that $G_{a}$ is a caloric function, integration by parts, (3.3) and the completion of the square of $\partial_{t} f+\frac{x}{2(t+a)} \cdot \nabla f-\frac{1}{2}\left(\Delta f+\partial_{t} f\right)$ yields the formula

$$
\begin{aligned}
\dot{D}_{a}(t) & =2 \int\left(\partial_{t} f+\frac{x}{2(t+a)} \cdot \nabla f-\frac{1}{2}\left(\Delta f+\partial_{t} f\right)\right)^{2} G_{a} d x \\
& -\frac{1}{2} \int\left(\Delta f+\partial_{t} f\right)^{2} G_{a} d x-D_{a}(t) /(t+a) .
\end{aligned}
$$


Then, from (3.2),(3.4) and the quotient rule

$$
\begin{aligned}
\dot{N}_{a}(t) & =\frac{4(t+a)}{H_{a}(t)^{2}}\left\{\int\left(\partial_{t} f+\frac{x}{2(t+a)} \cdot \nabla f-\frac{1}{2}\left(\Delta f+\partial_{t} f\right)\right)^{2} G_{a} d x H_{a}(t)\right. \\
& -\left(\int f\left(\partial_{t} f+\frac{x}{2(t+a)} \cdot \nabla f-\frac{1}{2}\left(\Delta f+\partial_{t} f\right)\right) G_{a} d x\right)^{2} \\
& \left.+\frac{1}{4}\left(\int f\left(\Delta f+\partial_{t} f\right) G_{a} d x\right)^{2}-\frac{1}{4} \int\left(\Delta f+\partial_{t} f\right)^{2} G_{a} d x H_{a}(t)\right\}
\end{aligned}
$$

and Lemma 6 follows from (3.5), the Cauchy-Schwarz inequality and the positiveness of the third term on the right hand side of (3.5).

The application of the Lemmas 6 and 2 to the proof of Theorem 1 is the following:

Lemma 7. Assume that $u$ satisfies, $\left|\Delta u+\partial_{t} u\right| \leq R^{2}|u|+R|\nabla u|,\|u\|_{\infty} \leq 1$, $\|\nabla u\|_{\infty} \leq R$ in $B_{4} \times\left[0, \frac{1}{R^{2}}\right]$ and $\|u(\cdot, 0)\|_{L^{2}\left(B_{1}\right)} d x \geq \theta R^{-n / 2}$ for some $\theta \in(0,1]$. Then, there is $N=N(n, \theta)$ such that

- $\left\|u(\cdot, 0) e^{-R^{2}|x|^{2} / \epsilon}\right\|_{L^{2}\left(B_{4}\right)} \geq e^{-N R^{2} \log \left(\frac{1}{\epsilon}\right)}$, when $0<\epsilon \leq \frac{1}{3 N}, R \geq N$.

- $\|u(\cdot, 0)\|_{L^{2}\left(B_{r}\right)} \geq e^{-N R^{2} \log \left(\frac{N}{r}\right)}$, when $0<r \leq \frac{1}{2}, R \geq N$.

Proof. The Lemma 1 with $\rho=1$ gives

$$
\sqrt{N}\|u(\cdot, t)\|_{L^{2}\left(B_{2}\right)} \geq R^{-n / 2} \text {, when } 0<t \leq \frac{1}{R^{2}} \text { and } R \geq N .
$$

Set $f=u \varphi$ in Lemma 6 , where $\varphi \in C_{0}^{\infty}\left(B_{4}\right), 0 \leq \varphi \leq 1, \varphi=1$ in $B_{3}$ and $\varphi=0$ outside $B_{\frac{7}{2}}$. From (3.6),

$$
H_{a}(t) \geq N^{-1} R^{-n}(t+a)^{-n / 2} e^{-1 /(t+a)}, \text { when } t+a \leq \frac{1}{R^{2}}, R \geq N .
$$

and

$$
\left|\Delta f+\partial_{t} f\right| \leq R^{2}|f|+R|\nabla f|+N R \chi_{B_{4} \backslash B_{3}}, \text { in } B_{4} \times\left[0, \frac{1}{R^{2}}\right] .
$$

From Lemma 6, (3.8) and (3.7), we have

$$
\dot{N}_{a}(t) \geq-N R^{2}-N R^{2} N_{a}(t), \text { when } t+a \leq \frac{1}{R^{2}}, R \geq N .
$$

Thus

$$
e^{N R^{2} t} N_{a}(t)+e^{N R^{2} t} \text { is nondecreasing, when } t+a \leq \frac{1}{R^{2}} \text { and } R \geq N .
$$

The multiplication of the identity

$$
\dot{H}_{a}(t)=2 \int f\left(\Delta f+\partial_{t} f\right) G_{a} d x+2 D_{a}(t)
$$

by $(t+a) / H_{a}(t),(3.8)$ and (3.7), imply that for some $N>0$,

$$
N_{a}(t) \leq N\left[1+(t+a) \partial_{t} \log H_{a}(t)\right], \text { when } 0 \leq t+a \leq \frac{1}{R^{2}} .
$$

Set $\beta=\frac{1}{R^{2}}$. Then, from (3.11), (3.10) and (3.7)

$$
\begin{aligned}
N_{a}(0) & \lesssim N_{a}(\beta / 4)+1 \lesssim 1+\int_{\beta / 4}^{\beta / 2} \frac{N_{a}(t)}{(t+a)} d t \lesssim 1+\int_{\beta / 4}^{\beta / 2} \partial_{t} \log H_{a}(t) d t \\
& =1+\log \left(\frac{H_{a}(\beta / 2)}{H_{a}(\beta / 4)}\right) \leq N R^{2}, \text { when } a \leq \frac{\beta}{12} .
\end{aligned}
$$


In particular,

$$
\begin{aligned}
& 2 a \int|\nabla f(x, 0)|^{2} e^{-|x|^{2} / 4 a} d x+\frac{n}{2} \int f^{2}(x, 0) e^{-|x|^{2} / 4 a} d x \\
& \quad \leq N R^{2} \int f^{2}(x, 0) e^{-|x|^{2} / 4 a} d x, \text { when } 0<a \leq \frac{1}{12 N R^{2}}, R \geq N .
\end{aligned}
$$

Now, Lemma 2 and (3.12) give

$$
\partial_{a} \log \left(\int f^{2}(x, 0) e^{-|x|^{2} / 4 a} d x\right) \leq \frac{N R^{2}}{a}, \text { when } 0<a \leq \frac{1}{12 N R^{2}}, R \geq N,
$$

and the integration of this inequality over $\left[\frac{\epsilon}{4 R^{2}}, \frac{1}{12 N R^{2}}\right]$ implies the first claim in Lemma 7. The second claim is derived from (3.12), as in Lemma 5.

Proof of Theorem 1. Proceeding as before, we may assume that $u$ satisfies

$$
\left|\Delta u+\partial_{t} u\right| \leq|u|+|\nabla u| \text { and }|u| \leq 1 \text { in } \mathbb{R}^{n} \times[0,4] .
$$

Choose then $\theta$ in $(0,1]$ such that, $\theta \leq\|u(\cdot, 0)\|_{L^{2}\left(B_{1}\right)}$ and set $u_{R}(x, t)=u(R x+$ $\left.y, R^{2} t\right)$, when $R=2|y|$ is large, $y \in \mathbb{R}^{n}$. Then,

$$
R^{n / 2}\left\|u_{R}(\cdot, 0)\right\|_{L^{2}\left(B_{1}\right)}=\|u(\cdot, 0)\|_{L^{2}\left(B_{2|y|}(y)\right)} \geq\|u(\cdot, 0)\|_{L^{2}\left(B_{1}\right)} \geq \theta
$$

and the arguments proceed as in the first proof.

\section{Proof of Theorem 2}

Theorem 2 follows with similar arguments. Here is an outline of its proof with a frequency function type argument .

Proof of Theorem 2. As before and without loss of generality we may assume that

$$
\left|\Delta u+\partial_{t} u\right| \leq|u|+|\nabla u| \text { and }|u| \leq 1 \text { in } \mathbb{R}_{+}^{n} \times[0,4] .
$$

Choose then $\theta$ in $(0,1]$ such that, $\theta \leq\|u(\cdot, 0)\|_{L^{2}\left(B_{1}\left(4 e_{n}\right)\right)}$. The argument in the proof of Lemma 1 (See also [7, Lemma 1]) is easily adapted to show that there is $N=N(n, \theta)>0$ such that

$$
\sqrt{N}\|u(\cdot, t)\|_{L^{2}\left(B_{2}\left(4 e_{n}\right)\right)} \geq 1, \text { when } 0<t \leq \frac{1}{N} .
$$

Set $v(x, t)=u\left(y x+y e_{n}, y^{2} t\right)$, when $y>8$. The fact that the ball of radius $2 / y$ and centered at $(4 / y) e_{n}-e_{n}$ is contained in $B_{1-2 / y}$, the change of variables, $z=y x+y e_{n}$ and (4.1), imply that

$$
\sqrt{N}\|v(\cdot, t)\|_{L^{2}\left(B_{1-2 / y}\right)} \geq y^{-n / 2}, \text { when } 0 \leq t \leq 1 /\left(N y^{2}\right) .
$$

Set $f=v \varphi$ in Lemma 6 , where $\varphi \in C_{0}^{\infty}\left(B_{1}\right), 0 \leq \varphi \leq 1, \varphi=1$ in $B_{1-1 / y}$ and $\varphi=0$ outside $B_{1-1 /(2 y)}$. From (4.2)

$$
H_{a}(t) \geq N^{-1} y^{-n}(t+a)^{-n / 2} e^{-\left(1-\frac{2}{y}\right)^{2} / 4(t+a)}, \text { when } t+a \leq \frac{1}{N y^{2}} .
$$

and

$$
\left|\Delta f+\partial_{t} f\right| \leq y^{2}|f|+y|\nabla f|+N y^{2} \chi_{B_{1} \backslash B_{1-1 / y}}, \text { in } B_{1} \times\left[0,4 / y^{2}\right]
$$


The calculations, which were carried out out in the second proof of Theorem 1 but replacing (3.7) and (3.8) by (4.3) and (4.4) respectively, imply the inequality

$$
\begin{aligned}
& 2 a \int|\nabla f(x, 0)|^{2} e^{-|x|^{2} / 4 a} d x+\frac{n}{2} \int f^{2}(x, 0) e^{-|x|^{2} / 4 a} d x \\
& \leq N y^{2} \int f^{2}(x, 0) e^{-|x|^{2} / 4 a} d x, \text { when } 0<a \leq \frac{1}{12 N y^{2}},
\end{aligned}
$$

which as seen before, implies the first part of Theorem 2. The second claim follows from the first, the results in [1] or [12, Theorem 3] and the qualitative result in [9], which was stated in the Introduction after Theorem 1.

\section{References}

[1] G. Alessandrini and S. Vessella, Remark on the strong unique continuation property for parabolic equations, Proc. Amer. Math. Soc. 132 (2003), no. 2, 499-501.

[2] J. Bourgain and C.E. Kenig, On localization in the continuous Anderson-Bernoulli model in higher dimension. To appear in Invent. Math.

[3] X.Y. Chen, A strong unique continuation theorem for parabolic equations, Math. Ann. 311 (1996) 603-630.

[4] L. Escauriaza, Carleman inequalities and the heat operator, Duke Math. J. 104 (2000), no. 1, 113-127.

[5] L. Escauriaza and L. Vega, Carleman inequalities and the heat operator II, Indiana U. Math. J. 50 (2001), no. 3, 1149-1169.

[6] L. Escauriaza and F.J. Fernández, Unique continuation for parabolic operators, Ark. Mat. 41 (2003) 35-60.

[7] L. Escauriaza, F.J. Fernández, and S. Vessella Doubling properties of caloric functions. Applicable Analysis 41 (2003) 35-60.

[8] L. Escauriaza, G. Seregin, and V. Šverák, Backward uniqueness for parabolic equations, Arch. Rational Mech. Anal. 169 (2003) 147-157.

[9] _ Backward uniqueness for the heat operator in half space, St. Petesburg Math. J. 15 (2004) 139-148.

[10] $\quad L^{3, \infty}$ - solutions to the Navier-Stokes equations and backward uniqueness, Russ. Math. Surv. 58 (2003) 211-250.

[11] L.C. Evans, Partial differential equations, Amer. Math. Soc., 1998.

[12] F.J. Fernández, Unique continuation for parabolic operators II, Comm. Part. Diff. Equat. 28 (2003), no. 9-10, 1597-1604.

[13] R. Hamilton, Monotonicity formulas for parabolic flows on manifolds, Comm. Anal. Geom. 1 (1993), no.1, 100-108.

[14] _ A matrix Harnack estimate for the heat equation, Comm. Anal. Geom. 1 (1993), no. $1,88-99$.

[15] L. Hörmander, Linear partial differential operators, Springer, 1963.

[16] - Uniqueness theorems for second order elliptic differential equations, Comm. Part. Diff. Equat. 8 (1983) 21-64.

[17] O.A. Ladyženskaja and V.A. Solonnikov, Linear and quasilinear equations of parabolic type, Translations of mathematical monographs, Amer. Math. Soc., 1968.

[18] E.M. Landis and O.A. Oleinik, Generalized analyticity and some related properties of solutions of elliptic and parabolic equations, Russian Math. Surv. 29 (1974) 195-212.

[19] F.H. Lin, A uniqueness theorem for parabolic equations, Comm. Pure Appl. Math. 42 (1988) $125-136$.

[20] S. Micu and E. Zuazua, On the lack of null-controllability of the heat equation on the half space, Port. Math. 58 (2001), no. 1, 1-24.

[21] C.C. Poon, Unique continuation for parabolic equations, Comm. Part. Diff. Equat. 21 (1996) $521-539$. 
[22] J.C. Saut and B.Scheurer, Unique continuation for evolution equations, J. Differential Equations 66 (1987), 118-137.

[23] G. Seregin, V. Šverák, The Navier-Stokes equations and backwards uniqueness, Nonlinear Problems of Mathematical Physics and Related Topics, Vol. 2, Kluwer Acad./Plenum Publ., 2002, pp. 359-370.

[24] C.D. Sogge, A unique continuation theorem for second order parabolic differential operators, Ark. Mat. 28 (1990) 159-182.

[25] F. Treves, Linear partial differential equations, Gordon and Breach, 1970.

Universidad del País Vasco / Euskal Herriko Unibertsitatea, Dpto. de Matemáticas, Apto. 644, 48080 Bilbao, Spain.

E-mail address: mtpeszul@lg.ehu.es

Department of Mathematics, University of Chichgo, 5734 S. University Avenue, ChichGo, Illinois 60637

E-mail address: kenig@math.uchicago.edu

Department of Mathematics, South Hall, Room 6607, University of California, Santa BARBARA, CA 93106

E-mail address: ponce@math.ucsb.edu

Universidad del País Vasco / Euskal Herriko Unibertsitatea, Dpto. De matemáticas, Apto. 644, 48080 Bilbao, Spain.

E-mail address: mtpvegol@lg.ehu.es 